\title{
LA DIMENSIÓN RURAL-URBANA EN UN CAMBIO LINGÜÍSTICO EN CURSO EN LA ENTONACIÓN DEL CATALÁN DE MENORCA ${ }^{1}$
}

\author{
Paolo ROSEANO ${ }^{\mathrm{a}, \mathrm{b}}$ / Ignasi MASCARÓc / Wendy ElVIRA-García ${ }^{\mathrm{d}}$ / \\ Ana Ma. FernándeZ PLANAS ${ }^{a}$ \\ Universitat de Barcelona ${ }^{a}$ / University of South Africab / Institut Menorquí d'Estudis ${ }^{c}$ / \\ Universidad Nacional de Educación a Distancia ${ }^{d *}$ \\ paolo.roseano@ub.edu / ipmascaro@gmail.com / welvira@flog.uned.es / \\ anamariafernandez@ub.edu
}

\section{Resumen}

Entre los varios factores sociales que pueden influir el cambio entonativo, la dimensión territorial solo se ha tenido en cuenta en un conjunto extremadamente limitado de estudios, mientras que el papel de otras variables (como la edad, el género o la clase social) se ha considerado más a menudo. Este trabajo estudia la entonación de las preguntas totales informativas de la localidad rural de Ferreries, en la isla de Menorca, y llega a la conclusión de que el cambio entonativo en curso en el cercano centro urbano de Ciutadella no afecta a la entonación de Ferreries. Este caso de estudio (que, de momento, es el único en el que se ha analizado el papel de la variable social "urbano/rural") demuestra, por lo tanto, que las zonas rurales tienden a ser más resistentes a la difusión de los cambios lingüísticos también en el ámbito entonativo.

\section{Palabras clave}

cambio lingüístico, entonación, urbano/rural, catalán

\footnotetext{
${ }^{1}$ Este trabajo se ha podido llevar a cabo gracias al proyecto de investigación FFI2015-64859-P (IP Ana Ma. Fernández Planas). Agradecemos a Núria Sintes Carreras su colaboración, que se concretó en la grabación y edición de los datos de Ferreries en el marco de su trabajo de final de grado (Sintes Carreras 2016).

* Laboratori de Fonètica, Universitat de Barcelona, Gran Via de les Corts Catalanes 585, 08007 Barcelona.
} 


\section{THE RURAL-URBAN DIMENSION IN A LINGUISTIC CHANGE IN PROGRESS IN THE INTONATION OF}

\section{MENORCAN CATALAN}

Abstract

Among the various social factors that can influence the intonational change, the territorial dimension has been taken into account only in a limited set of studies, whereas the role of other variables (such as age, gender or social class) has been studied more often. This work studies the intonation of information-seeking yes-no questions of the rural settlement of Ferreries, on the island of Menorca, and concludes that the intonational change in course in the nearby urban area of Ciutadella does not affect the intonation of Ferreries. This case study (which, for the time being, is the only one in which the role of the "urban/rural" social variable has been analyzed) shows, therefore, that rural areas are more resistant to diffusion of linguistic changes also in the field of intonation.

\section{Palabras clave}

language change, intonation, urban/rural, Catalan

\section{Introducción}

En comparación con otros tipos de cambio, los cambios de tipo fonético y fonológico suprasegmental -en concreto en el ámbito de la entonación- han recibido una atención escasa, a pesar de que en las últimas dos décadas haya ido aumentando el número de estudios que abordan esa cuestión. Además, la atención de la comunidad científica no se ha repartido de manera uniforme entre los diferentes tipos de cambio entonativo que se han descrito, ya que la mayoría de ellos se han centrado en fenómenos que se pueden atribuir al contacto de lenguas. Por añadidura, entre los varios factores sociales que pueden influir el cambio lingüístico, la dimensión territorial se ha tenido en cuenta solo en un conjunto extremadamente limitado de estudios, mientras que otras variables sociolingüísticas como la edad, el género o la clase social han recibido mucha más atención.

El objetivo de este trabajo, pues, es el de contribuir a la descripción de los fenómenos de cambio entonativo tratando un caso de estudio en el que, como se verá a continuación, la transformación que se observa no puede atribuirse al contacto de lenguas. Además, se incluye en el modelo la dimensión territorial (en concreto, el tipo 
de asentamiento urbano vs. rural) con el fin de comprobar qué papel puede desempeñar en la difusión del cambio.

El texto se estructura en cuatro secciones principales. En la primera de ellas, se resume el estado de la cuestión, tanto en relación con los estudios en general sobre el cambio entonativo como en relación con la entonación de la variedad de catalán hablada en la isla de Menorca. En la segunda sección, se detalla la metodología del estudio, tanto en términos de recogida de los datos cuanto en términos de técnicas de análisis. A continuación, el apartado dedicado a los resultados resume los hallazgos más destacados, que se discuten en el apartado final del estudio, dedicado justamente a la discusión y a las conclusiones.

\section{Estado de la cuestión}

\subsection{El cambio entonativo}

El cambio entonativo es un fenómeno muy poco estudiado, por dos razones. Por una parte, los estudios entonativos -sobre todo en el caso de las lenguas románicashan conocido su auge solo en las últimas tres décadas, por lo que se trata de una disciplina relativamente reciente en comparación con otras ramas de los estudios fónicos. Por otra parte, mientras que disponemos de información sobre las características segmentales de las lenguas desde hace muchos años, para la entonación empezamos a disponer de datos (tanto en forma de grabaciones como de transcripciones) desde hace poco tiempo. Eso significa que, mientras que se puede reconstruir diacrónicamente la evolución fónica segmental desde el latín clásico hasta las lenguas románicas, es imposible hacer lo mismo desde el punto de vista entonativo. En otras palabras, solo podemos estudiar los cambios entonativos en sincronía. ${ }^{2}$ Ese factor, junto al hecho de que el cambio lingüístico sea lento y que los estudios

\footnotetext{
${ }^{2}$ Con excepciones contadas, como las que se discuten en Pešková et al. (2012) y Roseano et al. (2015).
} 
entonativos constituyan una disciplina nueva, explica la escasez de estudios sobre el cambio entonativo.

Además de ser poco numerosos, los trabajos de este tipo suelen centrarse en casos de estudio en los que el cambio entonativo se puede explicar como efecto del contacto de lenguas. ${ }^{3}$ Pese a todo, los estudios sobre el cambio entonativo en contextos de contacto han permitido establecer una tipología de los mecanismos de acuerdo con los cuales la entonación se puede ver afectada en situaciones de contacto (Roseano et al. 2015). Los mecanismos en cuestión son la transferencia directa (los adultos que aprenden imperfectamente una $\mathrm{L} 2$ transfieren involuntariamente a esa $\mathrm{L} 2$ algunas de las características de la entonación de su L1), la fusión (los bilingües mezclan los patrones de las dos lenguas y desarrollan sistemas de entonación diferentes de los que presentan los monolingües en cada uno de los idiomas), y la acomodación (los hablantes de una variedad de $\mathrm{L}$ adoptan algunas de las características entonativas de la variedad de la misma $L$ hablada por otro grupo social.).

Los cambios entonativos documentados que no se pueden atribuir al contacto entre lenguas son extremadamente escasos y se limitan, básicamente, al fenómeno del uptalk en varios dialectos del inglés (Ching 1982; Guy et al. 1986; Allan 1990; Warren 2005; Sullivan 2011), a la entonación del japonés de Tokio (Inoue 1998) y al caso de Menorca que ha sido explorado por Mascaró \& Roseano (2015). Justamente a causa de su escasez, esos casos resultan sumamente interesantes. Casi todos ellos parten de una perspectiva sociolingüística y analizan cómo algunas variables sociales (fundamentalmente el género y la edad) influyen en el cambio entonativo. Sin embargo, ninguno de ellos toma en consideración otra variable social, de tipo geográfico, que es la diferencia entre hablantes urbanos y rurales. Este trabajo se propone, justamente, aportar información acerca del posible papel de esa variable en la difusión del cambio entonativo. Nuestra hipótesis, que se fundamenta en los axiomas clásicos de la dialectología (Chambers \& Trudgill, 1988: 29, entre otros) y de la sociolingüística (Labov

\footnotetext{
${ }^{3}$ Estudios de ese tipo son los de Vella (2003), Elordieta (2003, 2006), Parth \& Plag (2006), McMahon (2004) Mennen (2004), Colantoni \& Gurlekian (2004), O’Rourke (2005), Simonet (2008, 2011), Hickey (2008), Swerts \& Zerbian (2010), Fagyal (2010), Meisenburg (2011), Feldhausen (2011), Nokes \& Hay (2012), Benet et al. (2012), Boula de Mareüil et al. (2012), Pešková et al. (2012, 2013), Muntendam (2013), Sichel-Bazin et al. (2012a, 2012b), Gabriel \& Kireva (2014), Nance (2015), Roseano et al. (2015), Fernández Rei (2016), entre otros.
} 
$1983,1990,2007$, entre otros), es que los hablantes rurales, sobre todo los varones mayores (los llamados NORMs, es decir, non-mobile old rural men), son menos proclives al cambio entonativo, mientras que las mujeres urbanas serían las más propensas. Formalmente, por lo tanto, el conjunto de hipótesis es el siguiente:

Hp1: los hablantes rurales son menos propensos al cambio entonativo;

$\mathrm{Hp2}$ : entre los hablantes rurales, los menos propensos al cambio entonativo son los hombres mayores.

\subsection{La entonación del catalán de Menorca}

La entonación de las variedades catalanas de la parte occidental de Menorca se ha analizado en varios trabajos (sobre todo en Mascaró 1987; Vanrell \& Mascaró 2013), que concuerdan en describir las interrogativas absolutas informativas de esas hablas como caracterizadas por un patrón entonativo que presenta un descenso rápido de F0 en la última sílaba tónica del enunciado, seguido por un ascenso en las postónicas finales (Figura 1). La subida final es el rasgo entonativo que distingue las hablas de Menorca de otros dialectos del catalán, que también presentan interrogativas caracterizadas por un rápido descenso en la última tónica, al que sin embargo no sigue una subida de FO sino un mantenimiento del mismo en el rango bajo del hablante (Figura 2). Además, desde el punto de vista sintáctico, las interrogativas tradicionales del catalán de Menorca suelen presentar una estructura SVO y están encabezadas por la partícula átona que. 


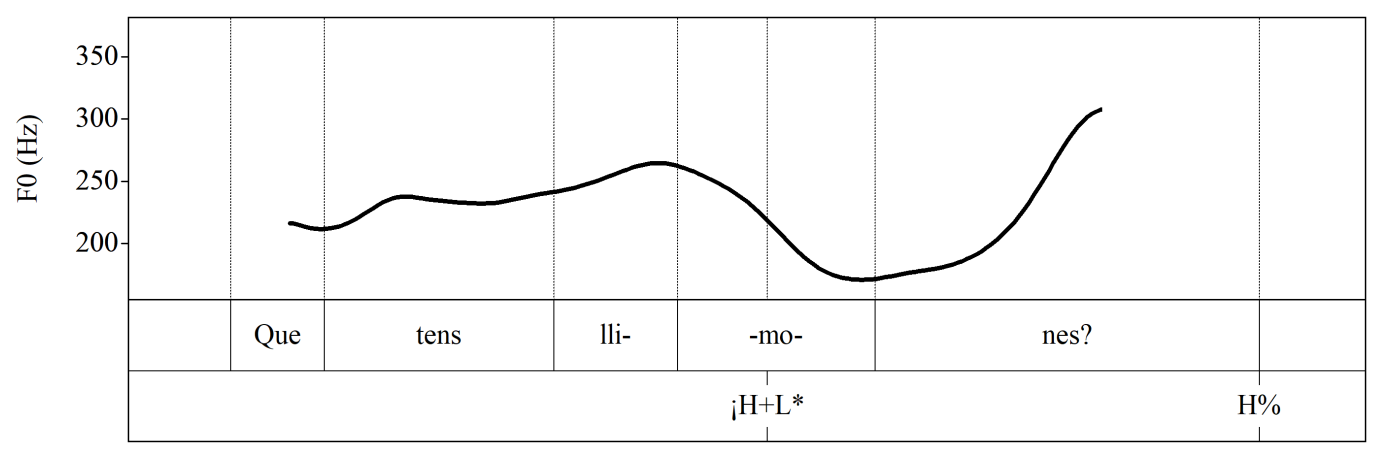

Figura 1. Contorno entonativo, transcripción ortográfica y anotación entonativa de la interrogativa absoluta neutra Que tens llimones? '¿Tienes limones?' pronunciada por un hablante de Ferreries (Menorca).

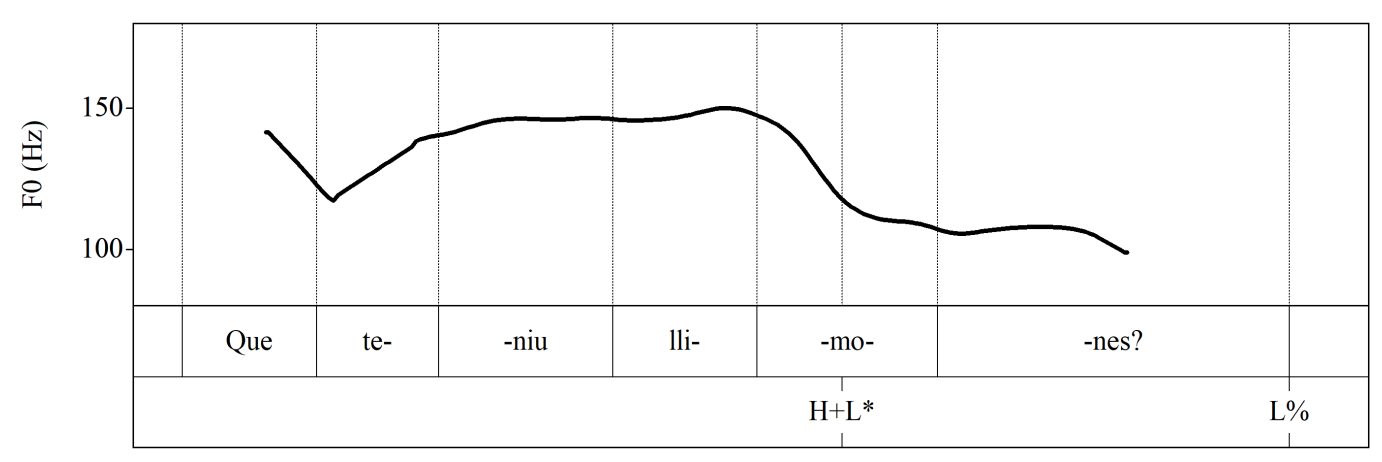

Figura 2. Contorno entonativo, transcripción ortográfica y anotación entonativa de la interrogativa absoluta neutra Que teniu llimones? ‘¿Tenéis limones?' pronunciada por un hablante de Barcelona.

Mascaró \& Roseano (2015) describen, para la ciudad de Ciutadella, una situación que se aleja de la que se ha resumido en el párrafo anterior. En su estudio, cuyos resultados se compararán detalladamente con los de este trabajo en el cuarto epígrafe, los autores demuestran que los adolescentes del asentamiento urbano en cuestión utilizan patrones entonativos que difieren de los de los adultos en dos aspectos. Por un lado, utilizan mayoritariamente un patrón de tipo ascendente-descendente (Figura 3) que en el repertorio entonativo tradicional de la isla solo aparece en las interrogativas reiterativas. Por otro lado, cuando utilizan el patrón tradicional, los adolescentes realizan con menos frecuencia el ascenso final que distingue el patrón interrogativo menorquín de los que aparecen en otros dialectos (Figura 4). 


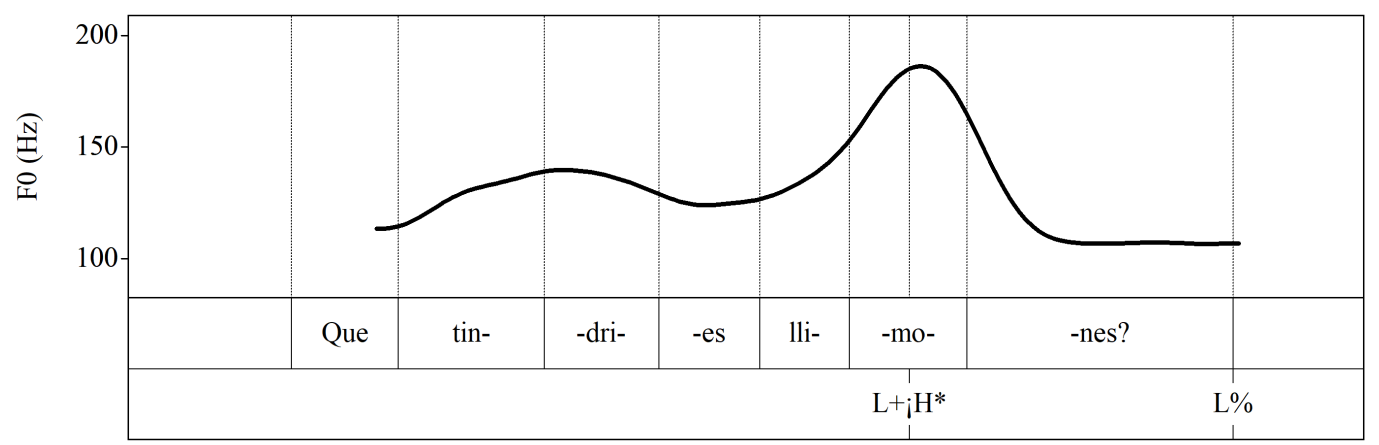

Figura 3. Contorno entonativo, transcripción ortográfica y anotación entonativa de la interrogativa absoluta neutra Que tindries llimones? '¿Tendrías limones?' pronunciada por un hablante de Ciutadella (Menorca).

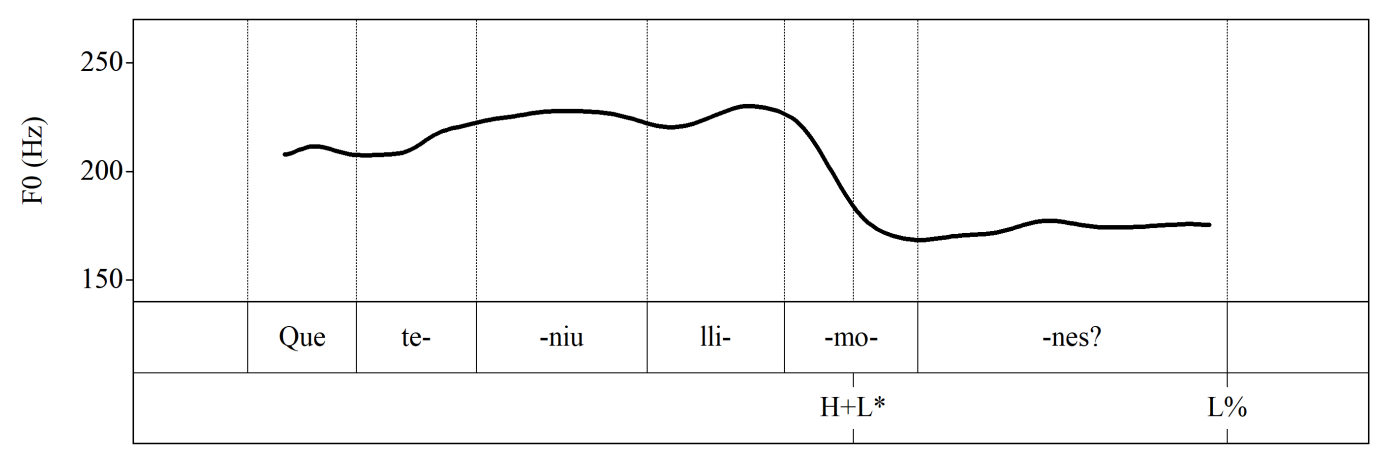

Figura 4. Contorno entonativo, transcripción ortográfica y anotación entonativa de la interrogativa absoluta neutra Que teniu llimones? '¿Tenéis limones?' pronunciada por un hablante de Ciutadella (Menorca).

Los autores dudan de que el patrón innovador sea el resultado del contacto de lenguas, ya que ninguna de las variedades románicas a las que están expuestos los adolescentes menorquines utiliza el patrón innovador, que ellos transcriben fonológicamente como $\mathrm{L}+\mathrm{iH}$ * $\mathrm{L} \%$, en las interrogativas absolutas: tanto en castellano peninsular central como en catalán central (y también en menorquín), de hecho, el patrón $\mathrm{L}+\mathrm{iH} \mathrm{H}^{*} \mathrm{~L} \%$ aparece solo en las preguntas no-neutras de tipo reiterativo ${ }^{4}$ (Estebas Vilaplana \& Prieto 2009; Borràs-Comes et al. 2014; Vanrell \& Mascaró 2013).

\footnotetext{
${ }^{4}$ En español peninsular central se utiliza en las interrogativas neutras otro patrón ascendentedescendente, que se transcribe $\mathrm{L}+\mathrm{H}^{*} \mathrm{~L} \%$ y que difiere de $\mathrm{L}+\mathrm{i} \mathrm{H}^{*} \mathrm{~L} \%$ en el rango, y esa diferencia es
} 
El estudio de Mascaró \& Roseano (2015) plantea también unas preguntas finales, que constituyen líneas de investigación futuras. Una de ellas está relacionada con el alcance geográfico de la innovación: los autores se preguntan si el cambio que observan en la ciudad de Ciutadella se observa también en otros puntos de la isla. Este trabajo tiene como primer objetivo justamente el de comprobar si el cambio que se observa en el núcleo urbano de Ciutadella también aparece en otro punto de la isla, que se caracteriza como asentamiento de tipo rural. A partir de los resultados obtenidos, se intentará reflexionar de forma más generalizada sobre la importancia de la dimensión urbano/rural en la difusión de los cambios lingüísticos.

\section{Metodología}

\subsection{Recogida de datos}

Los datos se han grabado en la isla mediterránea de Menorca, que forma parte del Archipiélago Balear. La población local habla una variedad de catalán que forma parte del dialecto balear, pero también utiliza el castellano, como lengua cooficial y de numerosos medios de comunicación. Por añadidura, gracias a los medios de comunicación, está expuesta también a otros dialectos del catalán (básicamente el catalán central y el mallorquín). Menorca presenta dos núcleos urbanos (Ciutadella, en el extremo occidental de la isla, y Maó, en la parte oriental), como una especie de doble capitalidad que tiene raíces históricas. Además de los dos centros urbanos, la ciudad presenta un conjunto de localidades rurales con menor población, algunas en la costa y otras en el interior.

Mientras Mascaró \& Roseano (2015) habían recogido sus datos en Ciutadella, que con sus más de 30.000 habitantes es el centro urbano principal de la isla, para este trabajo las grabaciones se han llevado a cabo en la primavera de 2016 en Ferreries, un

fonológica, tanto en castellano (Estebas Vilaplana \& Prieto 2009) como en catalán central (Borràs-Comes et al. 2014) y en catalán mallorquín (Mascaró \& Roseano 2015). 
pueblo más rural del interior de la isla, a unos $17 \mathrm{~km}$ de Ciutadella, cuya población asciende a poco más de 4.000 habitantes (Figura 5).

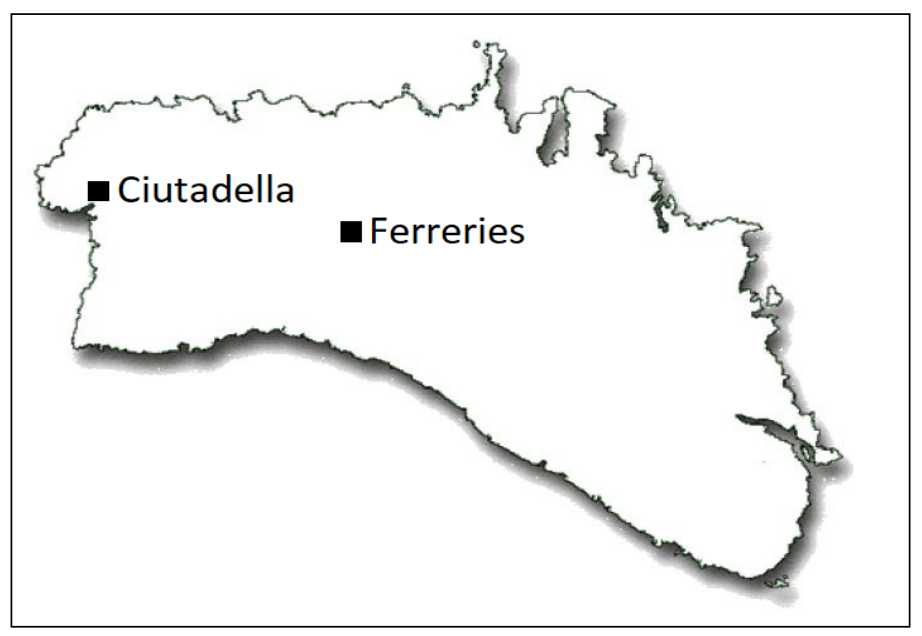

Figura 5. Mapa de la isla de Menorca con los dos puntos de encuesta que se citan en este trabajo (Ferreries y Ciutadella).

En Ferreries se contó con la colaboración de 47 informantes, cuya lengua habitual es el catalán en su variedad local. Todos ellos han vivido y nacido en Ferreries, no tienen estudios superiores y son de clase media. La muestra de entrevistados se ha estratificado por género y por generación. En cuanto a esta última variable, cabe destacar que se han clasificado los informantes en tres generaciones: adolescentes, jóvenes y adultos. La edad media de la generación de los adolescentes es de 15 años (con un mínimo de 12 y un máximo de 19); la de los jóvenes es de 22 años (con un mínimo de 20 y un máximo de 28); y la de los adultos es de 43 años (con un mínimo de 29 y un máximo de 60). En el conjunto, la división por sexo y generación de los informantes queda reflejada en la Tabla 1.

\begin{tabular}{|c|c|c|c|}
\hline & Mujeres & Hombres & Total \\
\hline Adolescentes & 8 & 8 & 16 \\
\hline Jóvenes & 8 & 7 & 15 \\
\hline Adultos & 7 & 9 & 16 \\
\hline Total & 23 & 24 & 47 \\
\hline
\end{tabular}

Tabla 1. Reparto de los informantes por generación y género. 
Tal y como habían hecho Mascaró \& Roseano (2015), los datos se obtuvieron mediante un cuestionario de situaciones, creado de acuerdo con el modelo del Discourse Completion Task (Blum-Kulka et al. 1989; Billmyer \& Varghese 2000; FélixBrasdefer 2010), habitual en las investigaciones sobre la prosodia, que consiste en plantear al informante una situación comunicativa de la vida cotidiana y pedirle que produzca la frase que utilizaría en ese contexto, como en el ejemplo (1).

(1) ENTREVISTADOR: Entras en una tienda en la que nunca habías entrado antes y preguntas si tienen limones.

ENTREVISTADO: ¿Tenéis limones?

La situación comunicativa del ejemplo (1) es la misma que se ha utilizado en casi todas las lenguas románicas para obtener preguntas totales informativas (Frota \& Prieto 2015). Para este trabajo se han utilizado tres variantes de la misma situación, en las que el hablante tenía que preguntar si en la tienda disponían de julivert 'perejil', llimones 'limones' y gírgoles 'setas de cardo'. De esta forma, se han podido elicitar frases acabadas en palabras agudas (julivert), llanas (Ilimones) y esdrújulas (gírgoles). Cada situación ha sido propuesta tres veces, alternadas con distractores. En total, por lo tanto, el número esperado de frase del corpus es 423 ( 3 situaciones $x 3$ repeticiones $\mathrm{x}$ 47 informantes). En realidad, sin embargo, el corpus que se analiza es de 409 frases porque algunas emisiones se han tenido que descartar, ya que los hablantes habían producido frases que, a pesar de ser correctas pragmáticamente en el contexto previsto, eran de otras modalidades (por ejemplo, interrogativas parciales como On és que hi ha gírgoles?, literalmente, ‘¿Dónde es que hay setas de cardo?’). La Tabla 2 ilustra de manera detallada el reparto de los ítems del corpus por género y generación. 


\begin{tabular}{|c|c|c|c|}
\hline & Mujeres & Hombres & Total \\
\hline Adolescentes & 72 & 70 & 142 \\
\hline Jóvenes & 72 & 54 & 126 \\
\hline Adultos & 63 & 78 & 141 \\
\hline Total & 207 & $\mathbf{2 0 2}$ & $\mathbf{4 0 9}$ \\
\hline
\end{tabular}

Tabla 2. Reparto de los ítems del corpus por generación y género.

\subsection{Análisis de datos}

Los datos se transcribieron entonativamente de acuerdo con los principios del sistema Cat_ToBI (Prieto et al. 2009). La transcripción se efectuó mediante el transcriptor automático Eti-ToBI (Elvira-García et al. 2016) y el resultado fue revisado, caso por caso, por el primer autor del artículo. De cada enunciado, se anotó prosódicamente la configuración nuclear (es decir, el conjunto del último acento tonal y de los tonos de frontera final). Para este estudio, las configuraciones relevantes son (i) $H+L^{*} H \%,(i) H+L^{*} L \%, L+i H^{*} L \%, y L^{*} H \%$. La primera de ellas, (i) $H+L^{*} H \%$, corresponde al patrón tradicional del menorquín y consiste en un descenso desde la última sílaba pretónica hasta la última tónica (a veces desde un tono extra-alto, de allí la presencia del diacrítico " $\mathrm{i}$ " entre paréntesis) y, a continuación, un ascenso en las sílabas siguientes hasta el final de la frase (Figura 1). El segundo, (i)H+L* L\%, es el mismo patrón, pero sin el ascenso final (Figura 4). El tercero, $\mathrm{L}+\mathrm{iH} * \mathrm{~L} \%$, es el patrón que Mascaró \& Roseano (2015) describen como patrón innovador y consiste en un ascenso de FO hasta un nivel extra-alto en la última sílaba tónica, seguido por un descenso hasta el final del enunciado. Finalmente, la configuración $L^{*} H \%$, que aparece solo marginalmente en los datos de Ferreries, corresponde al patrón ascendente que se ha descrito tradicionalmente tanto para el español peninsular central como para el catalán central, y que algunos autores ya han recogido en las variedades baleares del catalán (Simonet 2008, 2010; Szmidt \& Labraña 2013), a veces adscribiendo su presencia al efecto del contacto con el castellano (Simonet 2008: 180). 


\section{Resultados}

A primera vista, parece claro que los hablantes de Ferreries conservan muy mayoritariamente el patrón descendente, ya que este aparece -con o sin ascenso en las postónicas finales, es decir (i) $\mathrm{H}+\mathrm{L}^{*} \mathrm{H} \%$ o (i) $\mathrm{H}+\mathrm{L} * \mathrm{~L} \%$ - en un $88,8 \%$ de los casos (es decir, en 363 emisiones de 409). Los restantes casos están representados en un 7,3\% por patrones ascendente del tipo $L^{*} \mathrm{H} \%$ y en un 3,9\% por patrones innovadores del tipo $L+i H^{*} L^{*}$, el contorno que Mascaró \& Roseano (2015) describen como mayoritario entre los adolescentes de Ciutadella. Si comparamos los datos recogidos en Ferreries con los que Mascaró \& Roseano (2015) presentan para Ciutadella (Tabla 3), es evidente que en la localidad rural el patrón tradicional goza de una vitalidad que está perdiendo en el centro urbano. Esta diferencia urbano/rural, que constituye el foco principal de interés de este artículo, resulta significativa también desde el punto de vista estadístico (el test del Khi cuadrado proporciona $p<0,001)$.

\begin{tabular}{|l|c|c|c|}
\hline & $\begin{array}{c}\text { Patrones } \\
\text { tradicionales }\end{array}$ & $\begin{array}{c}\text { Patrones } \\
\text { nuevos }\end{array}$ & Total \\
\hline Ciutadella & 222 & 239 & 461 \\
\hline Ferreries & 363 & 46 & 409 \\
\hline
\end{tabular}

Tabla 3. Frecuencia de aparición de los patrones entonativos tradicionales (del tipo (i) H+L* $H \%$ ) y nuevos (de los tipos $L+i H^{*} L \%$ y $L^{*} H \%$ ) en Ciutadella (datos de Mascaró y Roseano, 2015) y en Ferreries.

Si consideramos los datos por generación (Tabla 4), observamos que los porcentajes en que aparecen los tres patrones entonativos en las tres generaciones son extremadamente parecidos, a pesar de que entre los adolescentes haya una tendecia ligeramente más alta a utilizar patrones no tradicionales: en conjunto, un $21,2 \%$ de los casos, frente al $4,8 \%$ de los jóvenes y al 7,1\% de los adultos. Si comparamos estos datos con los de Ciutadella, la diferencia está clara: Mascaró \& Roseano (2015) describen como ampliamente mayoritario (75,6\%) entre los adolescentes de Ciutadella el patrón innovador, mientras que los adultos utilizan de forma casi exclusiva $(98,0 \%)$ el patrón tradicional. 


\begin{tabular}{|l|c|c|c|c|}
\hline & $\mathbf{( i )} \mathbf{H + L} \mathbf{H} \%$ & $\mathbf{L}+\mathbf{i H} * \mathbf{L} \%$ & $\mathbf{L} * \mathbf{H} \%$ & Total \\
\hline Adolescentes & $78,9 \%$ & $9,2 \%$ & $12,0 \%$ & $100 \%(\mathrm{~N}=142)$ \\
\hline Jóvenes & $95,2 \%$ & $2,4 \%$ & $2,4 \%$ & $100 \%(\mathrm{~N}=126)$ \\
\hline Adultos & $92,9 \%$ & $0,0 \%$ & $7,1 \%$ & $100 \%(\mathrm{~N}=141)$ \\
\hline Total & $88,8 \%$ & $3,9 \%$ & $7,3 \%$ & $100 \%(\mathrm{~N}=409)$ \\
\hline
\end{tabular}

Tabla 4. Porcentajes de aparición de cada patrón entonativo por generación.

Si dividimos los datos por género (Tabla 5), tampoco observamos diferencias importantes en Ferreries, ya que ambos géneros mantienen muy mayoritariamente el patrón tradicional: en un $88,9 \%$ de los casos las mujeres; en un $83,8 \%$, los hombres.

\begin{tabular}{|l|c|c|c|c|}
\hline & $\begin{array}{c}\text { (i) } \mathbf{H}+\mathbf{L}^{*} \mathbf{H} \% \\
(\mathbf{i}) \mathbf{H}+\mathbf{L}^{*} \mathbf{L} \%\end{array}$ & $\mathbf{L + i H}$ * L\% & $\mathbf{L} * \mathbf{H} \%$ & Total \\
\hline Mujeres & $88,9 \%$ & $1,4 \%$ & $9,7 \%$ & $100 \%(\mathrm{~N}=207)$ \\
\hline Hombres & $88,6 \%$ & $6,4 \%$ & $5,0 \%$ & $100 \%(\mathrm{~N}=202)$ \\
\hline Total & $86,3 \%$ & $6,6 \%$ & $7,1 \%$ & $100 \%(\mathrm{~N}=409)$ \\
\hline
\end{tabular}

Tabla 5. Porcentajes de aparición de cada patrón entonativo por género.

Incluso si analizamos por separado solo las producciones de la generación de los adolescentes, tal y como hicieron Mascaró \& Roseano (2015), no observamos ninguna diferencia importante en relación con el género. Mientras en Ciutadella las mujeres adolescentes eran más propensas al cambio que sus coetáneos varones, en Ferreries los adolescentes de ambos géneros conservan el patrón tradicional en la misma medida, sin que las diferencias lleguen a ser significativas (75,0\% en las mujeres; $82,9 \%$ en los hombres). 


\section{Discusión}

En esta sección se discuten los resultados del análisis de los datos que se ha presentado en el apartado 4 con el fin de comprobar si las hipótesis que se han formulado en el apartado 2.1 son ciertas (sección 5.1). Además, se discute el interés que presenta la existencia, entre los hablantes de Ferreries, de dos patrones innovadores que compiten con el tradicional (sección 5.2).

\subsection{La variable urbano/rural en el cambio lingüístico}

Los resultados que hemos presentado permiten afirmar que la localidad rural de Ferreries es más resistente al cambio lingüístico que ya se está afirmando en el centro urbano de Ciutadella. Por lo tanto, en el ámbito del cambio entonativo, queda demostrada la Hp1, según la cual los grupos humanos en centros rurales serían más resistentes a las innovaciones, cuyo ámbito de origen suele ser urbano. Sin embargo, no hemos podido comprobar la Hp2 según la cual, dentro del ámbito rural, los hombres mayores serían el grupo menos propenso al cambio. Esa segunda hipótesis no se ha podido comprobar porque en Ferreries el cambio entonativo se encuentra en un estadio tan embrional que no parece aconsejable llegar a ningún tipo de conclusión a partir de los datos que se han recogido en este estudio.

\subsection{La competencia entre patrones innovadores}

Los datos de Ferreries difieren de los que recogieron Mascaró \& Roseano (2015) en Ciutadella en un aspecto que, potencialmente, plantea cuestiones muy interesantes de cara a la futura evolución de la entonación de esa variedad de catalán. Mientras Mascaró \& Roseano (2015) solo habían detectado dos patrones en las interrogativas absolutas de Ciutadella, en este trabajo se han encontrado tres. Además del patrón tradicional con sílaba nuclear descendente, los hablantes han utilizado dos contornos que no se han descrito en esa modalidad oracional del menorquín occidental: $L+i H^{*} L \%$ y L* H\%. Eso significa que habría dos patrones innovadores que pondrían en discusión el 
monopolio del contorno tradicional en el campo de las interrogativas absolutas neutras. Esa situación, en la que dos elementos innovadores compiten con uno tradicional por la misma función lingüística no se ha descrito nunca en los estudios sobre el cambio entonativo y habrá que esperar unos años para ver cómo se resuelve el conflicto replicando el estudio.

En ese sentido el caso de estudio de Ferreries parece extremadamente interesante y prometedor. Un seguimiento del caso en los próximos años podrá revelar qué dirección seguirá el cambio: podría asentarse el patrón innovador que Mascaró \& Roseano (2015) consideran autóctono $\left(\mathrm{L}+\mathrm{iH} \mathrm{H}^{*} \mathrm{~L} \%\right)$, podría afirmarse el patrón innovador que Simonet (2008) para el mallorquín atribuye al contacto con el castellano ( $\left.L^{*} H \%\right)$, podría observarse una reabsorción del cambio iniciado y el patrón tradicional $\left(i \mathrm{H}+\mathrm{L}^{*}\right.$ H\%) podría volver a ser el único, pero también podría pasar que los tres patrones (o dos de ellos) acabaran estabilizándose con funciones pragmáticas ligeramente diferentes.

\section{Conclusiones}

Este trabajo tenía el objetivo de proporcionar datos y realizar un análisis que arrojara luz sobre las dimensiones geográficas del cambio entonativo, un aspecto que hasta ahora no había sido explorado. El estudio de la entonación de las interrogativas totales informativas en la localidad de tipo rural de Ferreries, en la isla de Menorca, permite llegar a la conclusión de que la variable social "urbano/rural" es importante en los procesos de difusión de cambio entonativo, en la dirección que se podía hipotetizar a partir de los axiomas clásicos de la dialectología y la sociolingüística. Los hablantes de catalán de Ferreries, de hecho, han demostrado ser entonativamente más conservadores que los del centro urbano más cercano, Ciutadella, en el que entre los adolescentes se está difundiendo un patrón entonativo radicalmente diferente del tradicional. De esa manera, queda demostrado que, por lo menos en el caso de estudio descrito (que, de momento, es el único que ha tratado la variable en cuestión), también en el ámbito entonativo las zonas rurales son más resistentes a la difusión de los cambios lingüísticos. 
Aunque se haya podido comprobar que los hablantes rurales son entonativamente más conservadores, no se ha podido recoger ninguna prueba de que, entre los hablantes rurales, los menos proclives al cambio entonativo sean los varones mayores (los así llamados NORMs, es decir, non-mobile old rural men). Para poder confirmar o rechazar esa hipótesis, hará falta esperar a que el cambio entonativo se haya difundido intergeneracionalmente, momento que aún no ha llegado.

\section{Referencias bibliográficas}

AlLAN, Scott (1990) "The rise of New Zealand intonation", en A. Bell \& J. Holmes (eds.), New Zealand Ways of Speaking English, Clevendon: Multilingual Matters, 115-128.

BenET, Ariadna, Christoph GABRIEL, Elena KIREVA \& Andrea PEŠKOVÁ (2012) “Prosodic transfer from Italian to Spanish: Rhythmic properties of L2 speech and Argentinean Porteño", en M. Qiuwu, H. Ding \& D. Hirst (eds.), Proceedings of the 6th International Conference on Speech Prosody, Shanghai: Tongji University Press, 438-441.

BILLMYER, Kristine \& Manka VARGHESE (2000) "Investigating instrument-based pragmatic variability: Effects of enhancing discourse completion tests", Applied Linguistics, 21(4), 517-552.

BLUM-KULKA, Shoshana, Juliane HOUSE \& Gabriele KASPER (1989) "Investigating cross-cultural pragmatics: An introductory overview", en S. Blum-Kulka, J. House \& G. Kasper (eds.), Cross-cultural Pragmatics: Requests and Apologies, Norwood: Ablex, 1-34.

BorRÀs-COMES, Joan, Maria del Mar VanRell \& Pilar Prieto (2014) "The role of pitch range in establishing intonational contrasts", Journal of the International Phonetic Association, 44 (1), 1-20.

Boula de MAReüIL, Philippe, Albert Rilliard, Paolo MaIRANo \& Jean-Pierre LaI (2012) "Questions corses: peut-on mettre en évidence un transfert prosodique du corse vers le français?", en L. Besacier, B. Lecouteux \& G. Sérasse (eds.), Journées d'Etude sur la Parole (JEP 2012), Actes de la conférence conjointe JEP-TALN-RECITAL, Grenoble: Association Francophone pour la Communication Parlée (AFCP) y Association pour le Traitement Automatique des Langues (ATALA), vol. I, 609-616.

ChAMBers, Jack K. \& Peter Trudgill (1998) Dialectology, Cambridge: Cambridge University Press. CHING, Marvin K. L. (1982) "The question intonation in assertions", American Speech, 57, 95-107. 
ColAntonI, Laura \& Jorge GURLEKIAN (2004) "Convergence and intonation: Historical evidence from Buenos Aires Spanish", Bilingualism: Language and Cognition, 7, 107-119.

ELORDIETA, Gorka (2003) "The Spanish intonation of speakers of a Basque pitch-accent dialect", Catalan Journal of Linguistics, 2, 67-95.

ELORDIETA, Gorka (2006) "Spanish pitch accent alignment by Northern Bizkaian Basque speakers", en B. Fernández \& /I. Laka (eds.), Andolin gogoan. Essays in Honour of Professor Eguzkitza, Bilbao: University of the Basque Country, 269-290.

Elvira-García, Wendy, Paolo Roseano, Ana Ma. Fernández Planas \& Eugenio Martínez Celdrán (2016) "A tool for automatic transcription of intonation: Eti-ToBI a ToBI transcriber for Spanish and Catalan", Language Resources and Evaluation, 50 (4), 767-792.

Estebas Vilaplana, Eva \& Pilar Prieto (2009) “La notación prosódica en español. Una revisión del Sp_ToBI", Estudios de Fonética Experimental, XVIII, 263-283.

FAGYAL, Zsuzsanna (2010) Accents de banlieue: aspects prosodiques du français populaire en contact avec les langues de l'immigration, Paris: L'Harmattan.

Feldhausen, Ingo, Andrea PeŠKovÁ, Elena KIREVA \& Christoph Gabriel (2011) “Categorical perception of Porteño nuclear accents", en Proceedings of the 17th International Congress of Phonetic Sciences, 116-119.

$<$ https://www.slm.uni-hamburg.de/romanistik/personen/emeritiehemalige/gabriel/downloads/feldhausen-et-al-2011-categorical-perception-of-portenonuclear-accents.pdf>. [accessed 17.01.2018]

FÉLIX-BRASDEFER, J. César (2010) "Data collection methods in speech act performance: DCTs, role plays, and verbal reports", en A. Martínez-Flor \& E. Usó-Juan (eds.), Speech Act Performance: Theoretical, Empirical, and Methodological Issues, Amsterdam/Philadelphia: John Benjamins, 41-56.

FERNÁNDEZ REI, Elisa (2016) "Dialectal, historical and sociolinguistic aspects of Galician intonation", Dialectologia Special issue, VI, 147-169.

<http://www.publicacions.ub.edu/revistes/dialectologiaSP2016/>

FrotA Sónia \& Pilar PRIETO (eds.) (2015) Intonation in Romance, Oxford: Oxford University Press. INOUE, Fumio (1998) “Sociolinguistics of intonation change in progress in Tokyo", 東京外国語大 学論集 [Area and Culture Studies\}, 56, 11-24.

GABRIEL, Christoph \& Elena KIREVA (2014) "Prosodic transfer in learner and contact varieties: Speech rhythm and intonation of Buenos Aires Spanish and L2 Castilian Spanish produced by Italian native speakers", Studies in Second Language Acquisition, 36 (2), 257-281. 
GuY, Gregory R., Barbara HORVATH, Julia VonWILleR, Elaine DAISLEY \& Inge Rogers (1986) “An intonation change in progress in Australian English", Language in Society, 15, 23-52.

HICKEY, Raymond (2008) "Syntax and prosody in language contact and shift", en H. L. C. Tristram (ed.), The Celtic Languages in Contact. Papers from the Workshop within the Framework of the XIII International Congress of Celtic Studies, Bonn, 26-27 July 2007, Potsdam: Potsdam University Press, 235-244.

LABOV, William (1983) "Le changement linguistique - entretien avec William Labov", Actes de la Recherche en Sciences Sociales, 46, 67-71.

LABOV, William (1990) "The intersection of sex and social class in the course of linguistic change", Language Variation and Change, 2, 205-254.

LABOV, William (2007) "Transmission and Diffusion", Language, 83, 344-387.

MASCARÓ, Ignasi (1987) “Ciutadella-Maó. Greu vs. agut en dos parlars menorquins. Plantejament de la qüestió", Randa, 21, 197-211.

MASCARÓ, Ignasi \& Paolo RoSEANo (2015) “Un canvi entonatiu en curs: Les interrogatives absolutes entre els adolescents menorquins", Randa, 74, 139-153.

MCMAHON, April (2004) "Prosodic change and language contact", Bilingualism: Language and Cognition, 7, 121-123.

MeISENBURG, Trudel (2011) "Prosodic phrasing in the spontaneous speech of an Occitan/French bilingual", en C. Gabriel \& C. Lleó (eds.), Intonational Phrasing in Romance and Germanic: Cross-linguistic and Bilingual Studies, Amsterdam: John Benjamins, 127-151.

MENNEN, Ineke (2004) "Bi-directional interference in the intonation of Dutch speakers of Greek", Journal of Phonetics, 32, 543-563.

MunTENDAM, Antje G. (2013), "On the nature of crosslinguistic transfer: A case study of Andean Spanish", Bilingualism: Language and Cognition, 16, 111-131.

NANCE, Claire (2015) "Intonational variation and change in Scottish Gaelic", Lingua, 160, 1-19.

NOKES, Jacqui \&y Jennifer HAY (2012) "Acoustic correlates of rhythm in New Zealand English: A diachronic study", Language Variation and Change, 24, 1-31.

O'ROURKE, Erin (2005) Intonation and Language Contact: A Case Study of Two Varieties of Peruvian Spanish, Tesis doctoral, University of Illinois at Urbana-Champaign.

PARTH, Batt \& Ingo PLAG (eds.) (2006) Stress, tone and intonation in creole and contact languages, Special issue of Spachtypologie and Universalienforschung/Language Typology and Universals, 59 (2), 131-218.

PeŠKovÁ, Andrea, Ingo Feldhausen \& Christoph Gabriel (2013) “Una perspectiva diacrónica de la entonación bonaerense", en E. Casanova Herrero \& C. Calvo Rigual (eds.), Actas del XXVI 
Congreso Internacional de Lingüistica y de Filología Románicas, Berlin: De Gruyter, vol. I, 719-730.

PeŠKovÁ, Andrea, Ingo Feldhausen, Elena KIREVA \& Christoph Gabriel (2012) “Diachronic prosody of a contact variety: Analyzing Porteño Spanish spontaneous speech", en K. Braunmüller \& C. Gabriel (eds.), Multilingual Individuals and Multilingual Societies, Amsterdam: John Benjamins, 365-389.

PRIETo, Pilar, Lourdes AgUilar, Ignasi MASCARÓ, Francesc Josep TORReS \& Maria del Mar VANReLL (2009) "L'etiquetatge prosòdic Cat_ToBI", Estudios de Fonética Experimental, XVIII, 287309.

Roseano, Paolo, Ana Ma. Fernández Planas, Wendy Elvira-García \& Eugenio Martínez Celdrán (2015) “Contacto lingüístico y transferencia prosódica: El caso del alguerés”, Dialectologia et Geolinguistica, 23, 95-123.

SiCHEL-BAZIN, Rafèu, Carolin BUTHKE \& Trudel MeISENBURG (2012a) "Language contact and prosodic interference: Nuclear configurations in Occitan and French statements of the obvious", en M. Qiuwu, H. Ding \& D. Hirst (eds.), Proceedings of the 6th International Conference on Speech Prosody, Shanghai: Tongji University Press, vol. I, 414-417.

SiCHel-BAZIN, Rafèu, Carolin BUtHKE \& Trudel MeISEnBuRg (2012b) “The prosody of Occitan-French bilinguals", en K. Braunmüller \& C. Gabriel (eds.), Multilingual Individuals and Multilingual Societies, Amsterdam: John Benjamins, 349-364.

SIMONET, Miquel (2008) Language Contact in Majorca: An Experimental Sociophonetic Approach, Tesis doctoral, University of Illinois at Urbana-Champaign.

SIMONET, Miquel (2011) "Intonational convergence in language contact: Utterance-final contours in Catalan-Spanish bilinguals", Journal of the International Phonetic Association, 41, 185205.

SINTES CARRERAS, Núria (2016) Un canvi entonatiu en les interrogatives dels joves menorquins, Trabajo final de grado inédito, Universitat de Barcelona.

SULLIVAN, Jennifer N. (2011) Approaching Intonational Distance and Change, Tesis doctoral, University of Edinburgh.

SWERTS, Marc \& Sabine ZerBiAN (2010) "Prosodic transfer in Black South African English", en Proceedings of Speech Prosody 2010.

<http://speechprosody2010.illinois.edu/papers/100198.pdf>. (accessed 17.01.2018)

SZMIDT, Dorota T. \& Sabela LABRAÑA (2013) “Entonación de las oraciones interrogativas absolutas neutras en el catalán mallorquín", Onomázein, 27, 286-297. 
VanRelL, Maria del Mar \& Ignasi Mascaró (2013) "Balear", en P. Prieto \& T. Cabré (eds.), L'entonació dels dialectes catalans, Barcelona: Publicacions de l'Abadia de Montserrat, 75100.

VeLLA, Alexandra (2003) "Language contact and Maltese intonation: Some parallels with other language varieties", en K. Braunmüller \& G. Ferraresi (eds.), Aspects of Multilingualism in European Language History, Amsterdam: John Benjamins, 261-283.

WARREN, Paul (2005) "Patterns of late rising in New Zealand English: Intonational variation or intonation change?", Language Variation and Change, 17, 209-230. 